Post.Operative History.-Colon irrigations were started at once, and nutrient enemas were given, while feedings of water, albumin-water and whey were being slowly increased. Abdominal distention was considerable for several days, and the patient vomited twice, but nothing fecal. The distention was finally overcome by repeated enemas and stupes, together with the colon irrigations. The bowels moved spontaneously on the tenth day and convalescence was uneventful. A small amount of colon bacillus pus was evacuated from the upper angle of the wound, which thereupon healed kindly throughout. The patient was taken out of bed as soon as possible to avoid pulmonary hypostasis and was discharred from the hospital on the twentieth day after the operation. Since then she has been on regular diet, and has a normal bowel movement each day, the latter being assisted by the oceasional administration of liquid petrolatum, $1 / 2$ ounce. There is no abdominal distention and the femoral ring remains firmly elosed.

\section{CLINICAL REPORT OF A CASE OF RABIES TREATED WITH NEOSALVARSAN AND QUININ}

TOGETHER WITH A CASE OF LYSSOPHOBIA

\section{Miley B. Wesson, B.S., M.D., Eu Paso, Tex.}

The interest aroused by the publications of Tonin, ${ }^{1}$ Moon" and Harris $^{3}$ in the finding of a drug that would act as a specific for rabies warrants the publication of a report of this case, which was treated with both neosalvarsan and quinin.

C. M., Mexican, aged 10, was bitten, July 18, 1913, by a rabid dog, the left forearm and throat being badly lacerated; the wounds were cauterized and dressed daily at the charity clinic. Pasteur treatment was persistently urged but refused. About August 2 the boy became depressed and anxious and told the office girl at the clinic that he knew he was going mad. There were cephalalgia, loss of appetite accompanied by gastric uneasiness and nausea; the patient's mental eondition gradually grew worse and at times he was intermittently delirious. I saw him on the morning of August 6; the wounds were healed, but the scars appeared abnormally livid. The patient was restless, breathing was rasping and was frequently interrupted by pharyngeal and respiratory spasms; there was a slight dysphagia. A general hyperesthesia was present with photophobia and intolerance to sound. The eyes reacted to light and accommodation; tendon and skin reflexes were slightly exaggerated; the grip of the Jeft hand was much weaker than that of the right, and when the boy attempted to walk the left leg had a tendency to give way, so that he required support on that side.

I wrapped him in a blanket and carried him to the hospital in an open car (before permission for hospital treatment could be revoked by the family). The jolting of the car, the noises and the sunlight did not seem to bother him-in fact, the novelty of an automobile ride appeared to arouse him. As soon as he reached the hospital, however, he returned to his former lethargic condition.

Following the method of Tonin (who in July, 1912, reported a "hopeless" case of rabies in a 13-year-old child cured following the use of $0.3 \mathrm{gm}$. of salvarsan, potassium iodid, tepid baths and stimulants) this patient on admission to the hospital was given $0.4 \mathrm{gm}$. neosalvarsan (intravenously), repeated in twenty-four hours. On this day, Moon's report of rabies cured in dogs with quinin by mouth was received. So the patient was given quinin sulphate by mouth (106 grains per day, divided into three doses), and this was continued up to within a few hours of the end. During the course of his illness the temperature ranged from 100.2 to $106.2 \mathrm{~F}$., and pulse from 138 to 170

Forty hours before death he was unable to swallow egg-nog or other thick liquids, but he could swallow water up to

1. Tonin, R. : Policlinico, Rome, July 14, 1912.

2. Moon, V. HI. Jour. Infect. Dis., 1913, xifi, 1

3. Hirris, D. L. : A Clinical Report of Seven Cases of Hydrophobia, The Jovrxal A. M. A., Oct, 25, 1913, p. 1511. within four hours of lis death, though with great difficulty, suffocating pharyngeal spasms occurring after a few'swallows. The patient never became violent. Paralysis became general and death took place on the second day, from asphyxia during a slight paroxysm.

In this case neosalvarsan, quinin and other drugs had no influence in checking the advance of the disease or modifying its course in any way, other than possibly controlling convulsions; but if either neosalvarsan or quinin have this property possessed by none of the sedatives, then they are invaluable in the treatment of rabies.

\section{CASE OF LYSSOPIIOBIA}

Frequently cases of hysteria are diagnosed as hydrophobia, and when recovery occurs the cure is erroneously attributed to the last drug used. The following case is typical:

G. J. B., an Arizona mine-owner, was bitten on the right little finger, Nov. 11, 1913, while asleep, by a "hydrophobia skunk" (a small striped variety of skunk supposed by the cowboys to be always infected with rabies). He developed a pseudohydrophobia on the second day (though he had never seen a case of rabies) and paroxysms were so violent that he had to be restrained by his physician during the two days' trip to El Paso. I saw him Nov. 16, 1913; he was anxious, haggard looking and his eyes fairly blazed; there was difficulty in breathing and dysphagia was so marked that he had been practically without food or drink for two days; he was having frequent convulsions and appeared to have partially lost the use of his limbs. Severe pains radiated from the bitten finger to the back of his head.

Examination, made on admission to hospital, showed that. there was no paralysis, the superficial and deep reflexes were normal, and paroxysms, though violent, did not simulate those of rabies. The temperature was $97 \mathrm{~F}$. and the pulse 108. The patient was started on the Pasteur treatment, Negri bodies having been found in the skunk's brain. The convulsions were controlled by morphin, so suggestion instead of quinin or neosalvarsan was used and all symptoms disappeared. The patient was able to leave the hospital on the third day.

\section{A UNIQUE CASE OF BOWEL OBSTRUCTION}

W. D. Hamaker, M.D., Meadville, Pa.

History.-Mrs. P., aged 72, a patient of Dr. Best, has had fair health for many years with the exception of obstinate constipation. Nov. 1, 1913, she was seized with severe abdominal pain and nausea. Later on she had considerable distention and great tenderness over the abdomen. The tenderness was general and there was no resistance or tumefaction at any point. November 2 decided symptoms of obstruction showed themselves. High enemas given that day failed to produce much result, except one fairly large stool with some gas. Large doses of calomel and other cathartics had been given previously. Finally fecal vomiting appeared with hiccoughing.

Operation.-November 3 a laparotomy was performed. As the urine was almost entirely suppressed, November 2 and the morning of November 3, chloroform and oxygen were used. A median incision was made and the first thing noticed was a marked distention and conrestion of the small intestine. When the hand was passed in, deep in the cavity was found a mass, which, when drawn up, showed a black spot at one end. The mass proved to be Meckel's diverticulum pearshaped rolled up in one corner or edge of the omentum. The omentum tightly wrapped about and adherent to the diverticulum had produced complete gangrene. The findings, so far, would not account for the obstruetion of the bowels and on searching further I found a rent or hole through the upper part of the mesentery through which had passed all of the transverse colon as well as the omentum. There were no adhesions where the colon had passed through the mesentery but the condition appeared to be of long standing. This opening was the size of an egg and after the colon had been drawn up through the opening I closed it. The Meckel's 